\title{
A Proposed Framework for Security Aspects of Cloud Computing Services in Information Technology Companies
}

\author{
Amira Ahmed H. Tahon \\ MSc. Candidate \\ Sadat Academy \\ for Management Sciences
}

By

\author{
Prof. Dr. Farahat Farag Frahat, \\ Dept. of Computer and Information Systems, \\ Sadat Academy \\ for Management Sciences
}

\begin{abstract}
Undoubtedly failure to ensure proper security protection when using cloud services can ultimately lead to higher costs and potential losses of business and thus eliminate any potential benefits from cloud computing.

The aim of this research is to provide a practical reference to help IT companies in Egypt and business decision makers analyze the security implications of cloud computing on their business. The search included a list of steps, along with guidance and strategies, designed to help these decision makers evaluate and compare safety offerings of different cloud providers in key areas.

When thinking about moving to cloud computing, customers must have a clear understanding of the potential security benefits and risks associated with cloud computing, and develop realistic expectations with their cloud provider.
\end{abstract}

Keywords: Cloud Computing, Services Level Agreement, Security, Information Technology, Egyptian Companies

\section{1-Introduction}

Cloud Computing technology availability has become a fact in all lover the world, also it has become a fact in Egyptians it community since 2010.

This study explains the cloud computing types, advantage and its advantages, benefits and risks, some problems and solutions, security aspects and the most important element: services level agreement (SLA). Also the study in- troduces a proposed framework for security aspect of CC . Hoping that this study will help all interested community, especially in Egypt for better understanding awareness for this new trend, it may also help in pushing opening a great cloud market in Egypt, as well as speeding awareness of all aspects of cloud computing technology system, and dealing with its resources.

These resources can be dynamically configured and adjusted to a level that allows the user and the provider for the optimal utilization of resources. We are usually exploiting this gathering of resources by payment model versus use that offer full guarantees of any prior infrastructure provider. It must be obvious about security issues, before sensitive data, that it is important for cloud applications and transfer.

Cloud computing (CC) is a style of computing that delivers scalable functionality and flexible IT as a service to external customers using Internet technologies. It is not a revolutionary idea. Instead, it is an evolutionary concept that combines different existing technologies to provide new useful concepts for using existing technologies to outsource IT tool. Cloud computing (CC) can be seen to 3 layers, infrastructure-as-a service (IAAS), Platformas-a-service (PaaS), and software-as-a-service. Some examples of security issues and securing the physical network and servers, secure data storage, transfer, the cloud of integrity, accountability, flooding attacks, privacy 
and identity, authentication, browser security, and access management.

CC creates a Service Level Agreement (SLA), which is a contract between the user and the provider on the basis of legal liability and existing standards and addresses the unique challenges in the field of information technology legal liability standards. SLA emphasizes individuals and companies that leased the service that the cloud is a safe place for economic data. Thus, the Standard Service Level Agreement (SLA) that includes a «Bill of Rights» should be introduced.

Often using the default cloud computing for the exchange of resources and can provide the level of service to meet the client's quality of service (QoS) requirements. It is a hidden infrastructure detail that shows «Cloud» to the user as a single point of access to infrastructure.

The cloud is the beginning of the term that used to refer to the Internet and network systems where it was decided that the initial fee for the cloud is used to represent the transfer of data to the final position centers on the other side of the cloud.

The idea, as is the case when services through programs, «John McCarthy," a professor at Stanford University this idea, saying that «may regulate computing to a public become services in one day", where he saw that it was possible to perform the time - sharing technology (timesharing) to the future in terms of energy sold computing applications even as a service through a business model, and pointed out that the idea is already popular in the late sixties, but faded in the mid-seventies when it became abundantly clear that modern technology related to the field of IT is able to maintain this kind of future computing. But the idea has recently returned to become a common term in technological departments and institutions at the present time.

Emerged (CC conce again as one of the computing methods, which is to provide computing resources as services, and allows users to access the via the Internet (Cloud), without the need to acquire knowledge or experience, or even control of the infrastructure that supports these services.

As for the platforms of CC not work Amazon match EC2, Microsoft Azure Services Platform, Google App Engine, which give many companies the flexibility to access the necessary computing resources, and contribute to the reduction of the infrastructure of the emerging companies costs, but reliable is not without its problems, Many users raise issues such as privacy, security, etc ... when there is talk about the "cloud." So there is a growing interest in open source and cloud computing tools, which enable companies to build and customize computing «pull» to work alongside the most powerful business solutions.

\section{2-Research Goals and Objectives}

The main objective is to propose and achieve the standards from the standpoint of the security aspects of the agreements and the level of service for the adoption of CC in Egypt.

It aims to achieve the following objectives:

1. Intensive study covering the last CC applications centered in Egypt.

2. Current studies analysis of the use of CC services in Egypt.

3. Propose criteria for the adoption of CC in Egypt, which includes security issues.

Needs to be clear regarding the following:

- Leverage appropriate existing standards and to develop CC standards where gaps exist.

- How to make the balance between the benefits and risks, how to judge the legal issues which arise, and how contracts can maximize the advantages and minimize the disadvantages are other main objectives of the research.

- How to propose and analyze forecasts on the cloud which will greatly assist in making sure that the order to choose a cloud meets business objectives customers.

Therefore, the main aim of this research is to improve the understanding of SLA related practice and contents in C.C, rather than testing or confirming hypotheses involving specific research constructs.

\section{3 -Research Questions}

We will try to answer the following questions:

1. What is CC and what are the advantage and disadvantage of CC?

2. How to keep the balance between advantage and disadvantage of $\mathrm{CC}$ ?

3. What is services level agreement? And what should be standardized?

4. What are security problem and its solution?

5. What are security aspects in CC?

6. What is the proposed frame work for security aspects of $\mathrm{cc}$ ?

\section{4- Importance of research:}

The importance of research lies in the attention of companies and government to deploy CC technique in Egypt 
and through this conference which was held recently, and the most prominent event cloud week end conference, at Smart Village 16-17-May 2015 , Business Connect2014 conference events with a company IBM to introduce a whole new technological solution to protect the institutions data network CC. And the emphasis on the importance of $\mathrm{CC}$ technology, and giant databases and its role in government performance and the development of secure data. It is important to study the unclear legislative issues which come as a consequence of the dynamic handling of the cloud and its scalability. Cloud computing is a fundamental shift in IT as it enables IT system to be scalable and elastic. End users do not need to determine their exact computing resource requirements upfront.

The contract an organization will have with its (CCSP) shall be a key tool to help reduce its legal risks.

\section{5-Methodology}

The methodology that was followed in this research describes and discusses the research methods used for implementation. It begins with a description of the existing phenomena of CC. as well as the research design and approach, then, the discussion of the review of the literature.

This is followed by an explanation of the operations of the experimental data collection and data analysis. Finally, the last section contains a summary of the methodology.

\section{6-Research Evaluation Questionnaires and Results} of Analysis:

As the concept of CC has become one of the most important topics of discussion in the industry during the last period, therefore, the focus of this study will be to discuss this concept and the most important charge of it, and its effects on small and medium-sized companies in developing countries in general, and Egypt in particular in addition to the risks and challenges facing the transformation study, this new concept for the management of IT that came to know to see workers in data centers about their knowledge of $\mathrm{CC}$ and the benefits that accrue to the benefit of $\mathrm{CC}$ has been distributed to a number (32) of Forms. The general questionnaire constitutes of only 9 questions of general nature regarding Egyptian companies that are being surveyed. Appendix 2 constitutes 26 questions as related to SLA, Cost, Availability, Technical Support, and Security. Both appendices are being shown at the end of this paper. The Questionnaire 2 related to SLA, Cost, Availability, Technical Support, and Security has been analyzed according to the followings:
- SLA Question fromQ1-Q7

- Cost Questions from Q08-Q14,

- Availability Questions from Q15-Q16,

- Technical Support Questions from Q17-Q19, and

- Security Questions from Q20-Q26

Table 1: Result of the questionnaire 2 Questions.

\begin{tabular}{|c|c|c|c|c|c|c|c|c|c|c|}
\hline \multirow{2}{*}{ Variable } & \multicolumn{2}{|c|}{ Strongly agree } & \multicolumn{2}{|c|}{ Agree } & \multicolumn{2}{|c|}{ Neutral } & \multicolumn{2}{|c|}{ Disagree } & \multicolumn{2}{|c|}{ Strongly disagree } \\
\hline & Frequency & Percent & Frequency & Percent & Frequency & Percent & Frequency & Percent & Frequency & Percent \\
\hline Q1 & 18 & 47.40 & 12 & 31.60 & 2 & 5.30 & 0 & 0 & 0 & 0 \\
\hline$Q_{2}$ & 11 & 28.9 & 15 & 39.5 & 4 & 10.5 & 2 & 5.30 & 0 & 0 \\
\hline Q3 & 7 & 18.4 & 18 & 47.4 & 6 & 15.8 & 1 & 2.6 & 0 & 0 \\
\hline Q4 & 8 & 21.1 & 18 & 47.4 & 6 & 84.2 & 0 & 0 & 0 & 0 \\
\hline 0.5 & 5 & 13.2 & 19 & 50 & 5 & 13.2 & 3 & 7.9 & 0 & 0 \\
\hline Q6 & 6 & 15.8 & 19 & 50 & 4 & 10.5 & 2 & 5.3 & 1 & 2.6 \\
\hline Q7 & 15 & 44.1 & 8 & 23.5 & 6 & 17.6 & 3 & 8.8 & 0 & 0 \\
\hline Q8 & 17 & 50 & 9 & 26.5 & 5 & 14.7 & 1 & 2.9 & 0 & 0 \\
\hline$Q 9$ & 10 & 29.4 & 17 & 50 & 4 & 11.8 & 1 & 2.9 & 0 & 0 \\
\hline 010 & 6 & 17.6 & 13 & 38.2 & 6 & 17.6 & 6 & 17.6 & 1 & 2.9 \\
\hline Q11 & 6 & 17.6 & 13 & 38.2 & 6 & 17.6 & 6 & 17.6 & 1 & 2.9 \\
\hline Q12 & 6 & 17.6 & 16 & 47.1 & 7 & 20.6 & 3 & 8.8 & 0 & 0 \\
\hline Q13 & 4 & 11.8 & 20 & 58.8 & 4 & 11.8 & 3 & 8.8 & 1 & 2.9 \\
\hline Q14 & 11 & 34.4 & 18 & 56.2 & 1 & 3.1 & 2 & 6.2 & 0 & 0 \\
\hline Q15 & 5 & 15.6 & 17 & 53.1 & 8 & 25 & 2 & 6.2 & 0 & 0 \\
\hline 16 & 13 & 38.2 & 12 & 35.3 & 4 & 11.8 & 3 & 8.8 & 0 & 0 \\
\hline Q17 & 5 & 14.7 & 13 & 38.2 & 9 & 26.5 & 4 & 11.8 & 1 & 2.95 \\
\hline Q18 & 5 & 14.7 & 20 & 58.8 & 5 & 14.7 & 2 & 5.9 & 0 & 0 \\
\hline Q19 & 15 & 44.1 & 8 & 23.5 & 6 & 17.6 & 3 & 8.8 & 0 & 0 \\
\hline 020 & 17 & 50 & 9 & 26.5 & 5 & 14.7 & 1 & 2.9 & 0 & 0 \\
\hline Q21 & 10 & 29.4 & 17 & 50 & 4 & 11.8 & 1 & 2.9 & 0 & 0 \\
\hline Q22 & 6 & 17.6 & 13 & 38.2 & 6 & 17.6 & 6 & 17.6 & 1 & 2.9 \\
\hline Q23 & 6 & 17.6 & 13 & 38.2 & 6 & 17.6 & 6 & 17.6 & 1 & 2.9 \\
\hline Q24 & 6 & 17.6 & 16 & 47.1 & 7 & 20.6 & 3 & 8.8 & 0 & 0 \\
\hline Q25 & 4 & 11.8 & 20 & 58.8 & 4 & 11.8 & 3 & 8.8 & 1 & 2.9 \\
\hline Q26 & 15 & 44.1 & 8 & 23.5 & 6 & 17.6 & 3 & 8.8 & 0 & 0 \\
\hline
\end{tabular}

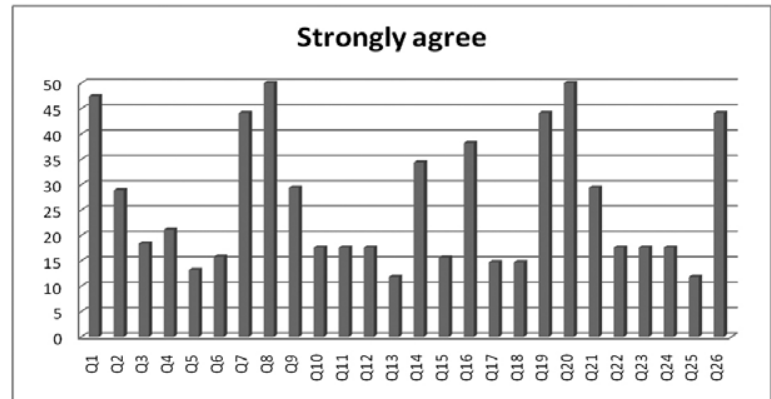

Figure (1)

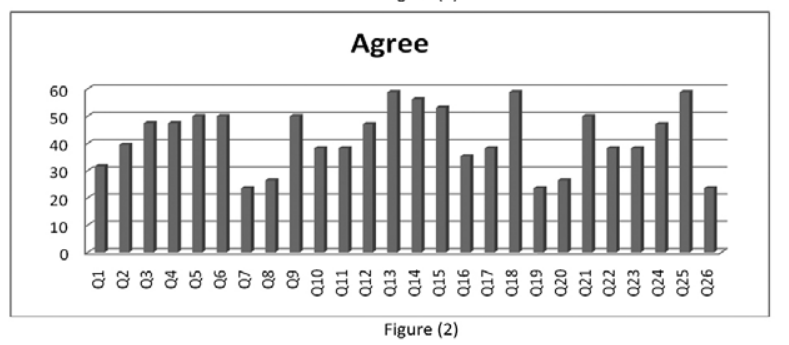

(a)

Neutral

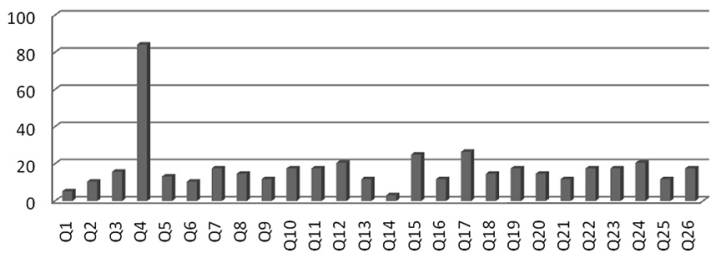

Figure (3)

7 (2) Compunet 22 (Jan - 2019)




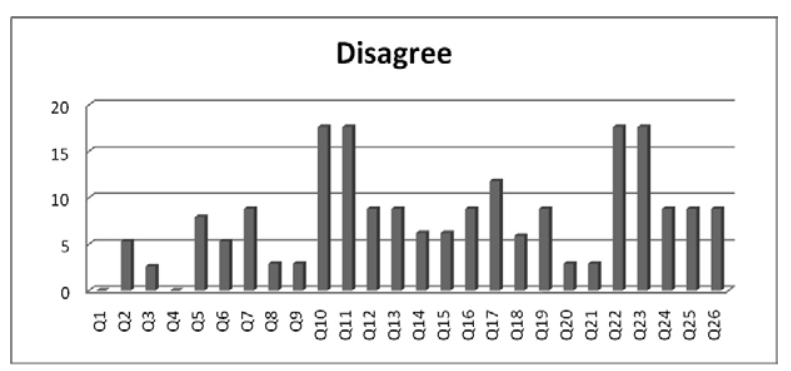

Figure (4)

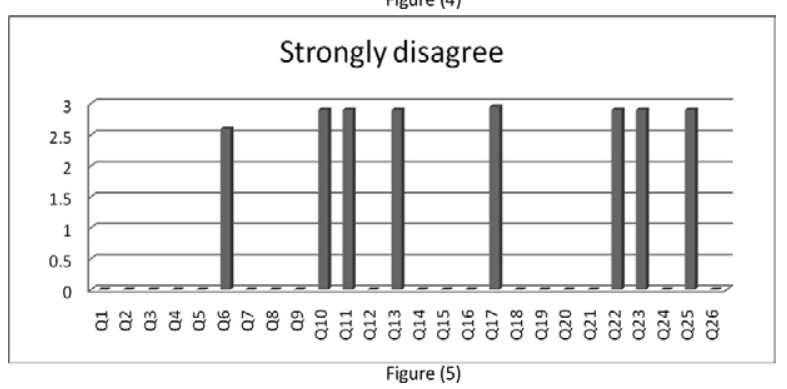

The Total variable of the Questions analysis are being indicated in the following table No. 2

Table (2) The Total variables

\begin{tabular}{|l|l|l}
\hline Variables & Reliability coefficient & Validity coefficient \\
\hline Tech. Support & 0.942 & 0.970 \\
\hline Available & 0.901 & 0.949 \\
\hline SLA & 0.947 & 0.972 \\
\hline COST & 0.970 & 0.984 \\
\hline Security & 0.973 & 0.986 \\
\hline All questions & 0.986 & 0.992 \\
\hline
\end{tabular}

The Main Results:

From figure (1),figure (2), figure (3), figure (4),figure(5) it is clear that:-

a high level of Reliability and Validity every variable has Reliability coefficients greater than $90 \%$, where the minimum Reliability coefficient we need is $60 \%$,so is the Reliability coefficient for all questions.

These same results are for Validity, where all Validity coefficients are greater than $90 \%$, so is the Validity coefficient for all questions.

This technique provides a survey of the statistical study of the extent of knowledge and understanding of cloud computing and applied to Businesses, government and individuals.

In this paper the researcher presented, a survey of a sample of the views of those interested in the field of CC and percentage of opinions.

\section{Conclusion}

Doing this research, the researcher answered the research questions related to the questionnaire (2) It was concluded that there are great obstacles like trust and reliance faces the service providers .In order to succeed in fulfilling all aspects of security, and attract more clients to use their services, great companies ( service providers) should overcome all these obstacles through some solutions: SLA and equivalent agreements should be introduced to control security of Data.

- SLA should be completed, upgrading and improving itself all the time.

- A respected and trust worthy valid international institutions (companies) should be untied instead of competing to reach the most reliable privacy policy in security.

- Companies must support the technologies and technicians to protect customer's application.

- Various providers should be available, so the customers can choose the best or favorites easily and confidently.

- Providers should seek, follow and ensure ISO/LEC2007 series of standard.

- All security agreements should be standardized

\section{Future Work}

For future work, we would like to go further in this topic, it will be really interesting to try to find and develop some secure application with Google APPS, or any other search engines.

In fact CC has the potential to become ornament safe and economically viable in the future of IT. We need much more technical and non-technical solutions to make the technology work and become more secure in our company. The concepts and some of the security aspects of cloud computing, we need more of the amendment here. Therefore, in the future we want to do more work in the area of security for the CC model as well as the security issues of privacy and the protection are the main challenges.

We propose in the future a set of management and protection of privacy frameworks unified identity across applications or cloud computing services. And audit data to the owners when they have access to the Data.

\section{9- Recommendations}

Companies need to know the options available to them and how they will deal with the cloud service provider of records management, in some cases, such dealing with the cloud service model to provide software as a service may be the integration of data retention and destruction with the offer provided by the service provider.

It should be on IT departments in companies to cooperate with the departments of Legal Affairs to identify the instructions and guidance on the extent of different cloud storage in many providers offer e-mail services software - also 
based on the development of policies for the service to delete e - mail messages after a full year.

In other cases, it is possible to keep the data through backup management software for companies, when evaluating cloud computing services, it is important to ask about the tools provided by the cloud service provider.

In some cases, the company will be able to use a combination of internal and external tools to facilitate the search for data identified. Also companies should face the multiple security threats like: Hijacking, loss of Governance or control or possibility of Data loss or leakage.

In another case when data is transferred to the cloud services, for example, you can change the ownership of the data and whether the company's rights in the various Data stored on the cloud service from those stored internally on its organs, so how can we evolutes and make sure of security aspects?

This process has become more complex when dealing with stored data on cloud services, and it requires painstaking effort before the signing of an additional contract with the cloud service provider.

Companies that you know whether you should re-locate data to the client in order to implement a legal or retain control, or is it possible to carry out legal processes Remember Me while they are in the cloud service. In addition, make sure the client not to take the cloud service provider from any action that would lead to the so - called tampering or destruction of evidence, which leads to the imposition of sanctions and the legal consequences resulting serious. Companies should be careful and cautious before the transfer of sensitive data to the cloud service because they may be affected by a harsh manner, Study sufficient to mitigate the risks of cloud services.

And we expect service providers to focus:

First: on reducing crashes common service during the application of policies and procedures to minimize the risk of privacy.

Second: protection, it is imperative that IT departments check the risk of transfer of data to the cloud services, at the same time, the share of small and large companies in the responsibility for ensuring the solution to all areas of interest and concern to ensure that the transition to a cloud service trade benefits and aggravate the burdens and legal responsibilities.

The development and formulation of service level agreements essential must be part of any negotiations with the cloud service provider. It must be clear rules on Data stored on the cloud service.

At the end, the last words, we can say that this newly appeared technology can be successful and well Spread in future, only if the providers of the services with the help of Governments make the necessary security Agreements, so that all right will be reserved For all parties.

List of Abbreviations\& Definitions

\begin{tabular}{|c|c|c|}
\hline NO & Abbreviations & Meaning \\
\hline 1 & Cloud SLAs & $\begin{array}{l}\text { Documented agreement between the cloud service provider and cloud } \\
\text { service customer that identifies services and cloud service level } \\
\text { objectives (SLOs). }\end{array}$ \\
\hline 2 & C.C & $\begin{array}{l}\text { Cloud Computing: } \\
\text { A paradigm for enabling network access to a scalable and elastic pool } \\
\text { of shareable physical or virtual resources with self-service } \\
\text { provisioning and administration on-demand. } \\
\text { Examples of resources include servers, operating systems, networks, } \\
\text { software, applications, and storage equipment. }\end{array}$ \\
\hline 3 & ISMS & Information Security Management Systems \\
\hline 4 & $\begin{array}{l}\text { Information } \\
\text { Security }\end{array}$ & $\begin{array}{l}\text { preservation of confidentiality, integrity and availability of } \\
\text { information; in addition, other properties, such as authenticity, } \\
\text { accountability, non-repudiation, and reliability can also be involved }\end{array}$ \\
\hline 5 & ISO 27001 & $\begin{array}{l}\text { The goal } \\
\text { - Provide the standard for Information Security Management Systems } \\
\text { Consists of } 11 \text { control sections, } 39 \text { control objectives, and } 133 \\
\text { controls } \\
\text { - Provide the base for third-party recognition } \\
\text { ISO } 27001 \text { Registrations/Certifications demonstrate conformance to } \\
\text { the standard }\end{array}$ \\
\hline 6 & SLA & service level agreement \\
\hline 7 & SLR & Systematic Literature Review methodology \\
\hline 8 & SMES & Small and Medium Enterprises \\
\hline 9 & SLM & Services level Management \\
\hline
\end{tabular}

\section{References:}

1 - Mell, P., \& Grance, T. (2011). The NIST definition of cloud computing,

page 2,3.

2 -Antonopoulos, N., \& Gillam, L. (2010). Cloud computing. London: Springer.2017_book,page 5, page 6.

3- Buyya, R., Ranjan, R., \& Calheiros, R. N. (2010, May). Intercloud: Utility-oriented federation of cloud computing environments for scaling of application services. In International Conference on Algorithms and Architectures for Parallel Processing (pp. 13-31). Springer, Berlin, Heidelberg.

4- Kwofie, B. (2013). Cloud computing opportunities, risks and challenges with regard to Information Security in the context of developing countries: A case study of Ghana,page 19 .

5- Buyya, R., Ranjan, R., \& Calheiros, R. N. (2010, May). Intercloud: Utility-oriented federation of cloud computing environments for scaling of application services. In International Conference on Algorithms and Architectures for Parallel Processing (pp. 13-31). Springer, Berlin, Heidelberg.page 14,page 15.

6-Boillat, T., \& Legner, C. (2013). From on-premise soft- 
ware to cloud services: the impact of cloud computing on enterprise software vendors, business models. Journal of theoretical and applied electronic commerce research, 8(3), 39-58.

7- Sabi, H. M., Uzoka, F. M. E., Langmia, K., \& Njeh, F. N. (2016). Conceptualizing a model for adoption of cloud computing education. International Journal of Information Management, 36(2), 183-191.

8-El Hadi, M. M. (2012). The Move of Business Enterprises Towards Using Cloud Computing], Egyptian Information Magazine EGYPT INFO, No.12, pp.26-32.

\section{2-References From Internet}

1 Jiménez Martínez, D. (2013). Privacy and confidentiality issues in cloud computing architectures (Master's thesis, Universitat Politècnica de Catalunya).

2 Mann, Z. A. (2018). Data protection in fog computing through monitoring and adaptation. KuVS-Fachgespräch Fog Computing 2018, 25

3 Chen, D., \& Zhao, H. (2012, March). Data security and privacy protection issues in cloud computing. In Computer Science and Electronics Engineering (ICCSEE), 2012 International Conference on (Vol. 1, pp. 647-651). IEEE.

4 Buckholtz, B., Ragai, I., \& Wang, L. (2015). Cloud manufacturing: current trends and future implementations. Journal of Manufacturing Science and Engineering, 137(4), 040902.

\section{Appendix (1) General Questionnaire}

Questionnaire:

1- Enter the name for your enterprise?

2- Enter the website for your enterprise?

1- Government and nonprofits

2- advertising / marketing /media

3- Travel and leisure

4- Healthcare

5 -other type

3- What is size of the enterprise you represent?

1- 10-49 employees (small enterprise)

2- 50-250 employees(medium enterprise)

3- Over 250 employees (medium enterprise

4- What is profile job title?

1- IT

2- NO IT

5- Which solutions does your enterprise use ?

1- Public cloud computing

2- Private cloud computing

3-Hybrid cloud computing

6- Which types of services are applied in enterprise ?

1- Saas software-as-a services
2- Paas platform as a services

3- laas infrastructure as a services

4- Security services in the cloud

5- Other

7- Which cloud providers are you dealing with ?
1- Amazon
2- Google
3- IBM
4- Microsoft
5- Rackspace
Other type

8 -what are the key barriers in cloud computing at enterprise?

1- Security

2- Confidentiality

3- Cost

4- Internet speed

Other

9- Who wrote the SLA?

1-service provider

2 - Customer

3 -both

4 - Third party... Specify

5- Other type

Appendix (2) Questions concerning SLA, Cost, Availability, Technical Support and Security

\section{SLA Questions:}

Q1: SLA is one of the most important criteria you have to use in evaluating cloud computing services.

1- Strongly agree, 2- Agree, 3- Neutral, 4- Disagree, 5Strongly. Disagree

Q2: The SLA provides a list of all services provided by the cloud provider with a full definition of each services

1- Strongly agree, 2- Agree, 3- Neutral, 4- Disagree, 5Strongly disagree

Q3: You are satisfied with the achievement of security condition mentioned in the SLA.

1- Strongly agree, 2- Agree, 3- Neutral, 4- Disagree, 5Strongly Disagree.

Q4: You are satisfied with the achievement of availability condition mentioned in the SLA.

1- Strongly Agree, 2- Agree, 3- Neutral, 4- Disagree, 5Strongly Disagree.

Q5: You are satisfied with the achievement of support condition mentioned in the SLA.

1- Strongly Agree, 2- Agree,, 3- Neutral, 4- Disagree, 5- Strongly Disagree>

Q6: You are satisfied with the achievement of cost condition mentioned in the SLA.

1- Strongly Agree, 2- Agree, 3- Neutral, 4- Disagree, 5Strongly Disagree. 
Q7: Is the user informed and aware of SLA and knowledge of the rights and the obligations between the parties.

1- Strongly Agree, 2- Agree, 3- Neutral, 4- Disagree, 5Strongly Disagree

\section{Cost Questios:}

Q8: Cloud computing reduces capital expenses (like the cost of Buying a data center in your enterprise.

1- Strongly agree 2- Agree 3-Neutral, 4- Disagree 5Strongly disagree

Q9: Cloud computing reduces cost of buying new software licenses in your enterprise.

1- Strongly agree, 2- Agree, 3- Neutral , 4- Disagree, 5- Strongly disagree

Q10: The cloud elasticity reduces the cost of using cloud computing

1- Strongly agree, 2- Agree, 3- Neutral, 4- Disagree 5Strongly disagree

Q11: The cloud provides complete security for sensitive data.

1- Strongly agree, 2-Agree, 3- Neutral , 4- Disagree. 5Strongly Disagree

Q12: When using data backup service, the data is secured enough.

1- Strongly Agree 2- Agree 3- Neutral 4- Disagree 5Strongly disagree

Q13: The connection used for data transmission is encrypted enough.

1- Strongly agree 2- Agree 3- Neutral 4- Disagree

5- Strongly disagree

Q14: The cloud protected enough all your data in case of natural disasters.

1- Strongly agree 2- Agree 3- Neutral 4-Disagree 5Strongly disagree

\section{Availability Questins}

Q15: Your service level of availability is appropriate for the nature of work and The type of used application in your enterprise

1- Strongly agree 2- Agree 3- Neutral 4- Disagree 5Strongly Disagree.

Q16 Its schedule cloud services to consumers by service providers affects in the Costs and benefits are challenges of cloud

1- Strongly agree 2-Agree 3- Neutral 4- Disagree5Strongly disagree

\section{Technical Support Questions}

Q17: The diversity (in support channels ) has a big role in the evaluation of the Cloud

1- Strongly agree 2- Agree 3- Neutral 4- Disagree 5-Strongly disagree

Q18: What are the support channels provided to your enterprise?
1- Strongly agree

2- Agree 3- Neutral 4-Disagree 5-Strongly disagree

Q19: The level of support provided is suitable to nature of work.

1- Strongly agree 2- Agre 3- Neutral 4-Disagre 5-Strongly disagree

\section{Security Questions}

Q20: The cloud provides complete security for sensitive data

1- Strongly agree, 2- Agree, 3- Neutral, 4-Disagree, 5-Strongly disagree.

Q21: When using data Backup service, the data is secured enough.

1-Strongly agree 2- Agree 3- Neutral 4-Disagree 5-Strongly disagree

Q22: The connection used for data transmission is encrypted enough.

1-Strongly Agree, 2- Agree, 3- Neutral, 4-Disagree, 5-Strongly disagree

Q23: The cloud protects enough all your data in case of natural disasters.

1-Strongly agree, 2- Agree, 3- Neutral, 4-Disagree, 5-Strongly Disagree.

Q24: The main security benefits of cloud computing are the improved Security software mechanisms and security maintenance.

1-Strongly Agree, 2- Agree, 3- Neutral, 4-Disagree, 5-Strongly Disagree.

Q25: Cloud providers have the capacity to develop more sophisticated security software. In addition, cloud customers, especially of SaaS, do not have to care about the security maintenance as the entire maintenance is executed by provider. Besides, they get the latest security software updates automatically

1-Strongly Agree, 2- Agree, 3- Neutral, 4-Disagree, 5-Strongly Disagree.

Q26: Among the security benefits of cloud computing for organizations are the lower financial security commitments and faster data recovery. Cloud providers perform data recovery much faster than companies that do not use cloud services.

1-Strongly Agree, 2- Agree, 3- Neutral, 4-Disagree, 5-Strongly Disagree

Q27: From cloud computing security risk is the confidential data and data loss. Organizations with which the confidential data does not host sensitive data in the cloud. These organizations hide large companies and non-commercial organizations.

1- Strongly agree 2- Agree 3- Neutral 4-Disagree 5-Strongly disagree 